TRANSACTIONS OF THE

AMERICAN MATHEMATICAL SOCIETY

Volume 354, Number 5 , Pages 1793-1809

S 0002-9947(02)02932-X

Article electronically published on January 10, 2002

\title{
ASYMPTOTIC LINEAR BOUNDS FOR THE CASTELNUOVO-MUMFORD REGULARITY
}

\author{
JÜRGEN HERZOG, LÊ TUÂN HOA, AND NGÔ VIÊT TRUNG
}

\begin{abstract}
We prove asymptotic linear bounds for the Castelnuovo-Mumford regularity of certain filtrations of homogeneous ideals whose Rees algebras need not be Noetherian.
\end{abstract}

\section{INTRODUCTION}

In a pioneering paper, Bertram, Ein and Lazarsfeld [BEL] proved that if $X \subset \mathbb{P}^{r}$ is a smooth complex variety of codimension $s$ which is cut out scheme-theoretically by hypersurfaces of degree $d_{1} \geq \ldots \geq d_{m}$, then $H^{i}\left(\mathbb{P}^{r}, \mathcal{I}_{X}^{n}(a)\right)=0$ for $i \geq 1$ and $a \leq d_{1} n+d_{2}+\cdots+d_{s}-r$. Their result has initiated the study of the CastelnuovoMumford regularity of the powers of a homogeneous ideal. The first result in this direction is due to Chandler [Ch] and Geramita, Gimigliano and Pitteloud [GGP]. They proved that if $I$ is a homogeneous ideal in a polynomial ring $R$ (over a field) with $\operatorname{dim} R / I \leq 1$, then $\operatorname{reg}\left(I^{n}\right) \leq \operatorname{reg}(I) n$ for all $n \geq 1$. This result does not hold for higher dimension, due to an example of Sturmfels [St]. However, Swanson [Sw] showed that $\operatorname{reg}\left(I^{n}\right)$ is always bounded by a linear function of the form $c n+e$. She could not provide an estimate for $c$. For monomial ideals, there were some attempts to estimate $c$ in terms of better understood invariants of $I$ (see [SS], [HT]). This problem seemed to be hard. So it came as a surprise that we can always choose $c \leq d(I)$, where $d(I)$ denotes the maximum degree of the generators of $I$, and that $\operatorname{reg}\left(I^{n}\right)$ is in fact a linear function for all large $n$.

Theorem 0.1. [CHT Theorem 3.1], $\mathrm{K}$, Theorem 5] Let I be an arbitrary homogeneous ideal in a polynomial ring. There exist constants $c \leq d(I)$ and $e \geq 0$ such that for all large $n$,

$$
\operatorname{reg}\left(I^{n}\right)=c n+e .
$$

The reason for this phenomenon comes from the fact that the Rees algebra $R[I t]=\bigoplus_{n \geq 0} I^{n} t^{n}$ is a Noetherian bigraded algebra. In fact, it can be shown that if $E=\bigoplus_{n \geq 0} E_{n}$ is a finitely graded bigraded module over the Rees algebra $R[I t]$, then $\operatorname{reg}\left(E_{n}\right)$ is a linear function of $n$ for all large $n$ (see CHT, Lemma 3.3 and Theorem 3.4]).

Recently, Cutkosky, Ein and Lazarsfeld proved the following related, more geometric result.

Received by the editors November 25, 2000.

2000 Mathematics Subject Classification. Primary 13D45.

Key words and phrases. Castelnuovo-Mumford regularity, reduction number, $a$-invariant, ideal.

The second and third authors are partially supported by the National Basic Research. 
Theorem 0.2. CEL, Theorem 3.2] Let I be an arbitrary homogeneous ideal in a polynomial ring. Let $\left(I^{n}\right)^{\text {sat }}$ denote the saturation of $I^{n}$. Then $\lim _{n} \frac{\operatorname{reg}\left(\left(I^{n}\right)^{\text {sat }}\right)}{n}$ exists.

Question 1. Let $(R, \mathfrak{m})$ be a local ring and $I$ an ideal in $R$. Let $G\left(R / I^{n}\right)$ denote the associated graded ring of $R / I^{n}$ with respect to $\mathfrak{m}$. Then $G\left(R / I^{n}\right)$ can be represented as a quotient ring of the associated graded ring $G(R)$ by a homogeneous ideal $I_{n}^{*}$, where $\left\{I_{n}^{*}\right\}$ is a filtration of ideals. Does $\lim _{n \rightarrow \infty} \frac{\operatorname{reg}\left(G(R) / I_{n}^{*}\right)}{n}$ exist?

Question 2. Let $R$ be a polynomial ring and $I$ a homogeneous ideal in $R$. Let $I^{(n)}$ denote the $n$th symbolic power of $I$. Does $\lim _{n \rightarrow \infty} \frac{\operatorname{reg}\left(I^{(n)}\right)}{n}$ exist?

Question 3. Let $R$ be a polynomial ring and $I$ a homogeneous ideal in $R$. Let in $(I)$ denote the initial ideal of $I$ with respect to an arbitrary term order. Does $\lim _{n \rightarrow \infty} \frac{\operatorname{reg}\left(\operatorname{in}\left(I^{n}\right)\right)}{n}$ exist?

In all these cases, the underlying bigraded "Rees algebra" need not be Noetherian; hence the method of [CHT] and [K] could not be applied directly. In fact, we do not know any general approach and any definite answer (yes or no) to these problems.

One may weaken the above questions by asking if the Castelnuovo-Mumford regularities $\operatorname{reg}\left(G\left(R / I_{n}\right)\right), \operatorname{reg}\left(I^{(n)}\right)$ and $\operatorname{reg}\left(\operatorname{in}\left(I^{n}\right)\right)$ are bounded by linear functions of $n$. In this paper we will prove the following results which suggest that the weakened questions may have a positive answer:

1. $\operatorname{reg}\left(G\left(R / I^{n}\right)\right)$ is bounded by a linear function if $\operatorname{dim} R / I \leq 1$.

2. $\operatorname{reg}\left(I^{(n)}\right)$ is bounded by a linear function in the following cases:

(i) $\operatorname{dim} R / I \leq 2$ (already discovered by Chandler [Ch]),

(ii) The singular locus of $R / I$ has dimension $\leq 1$,

(iii) $I$ is an arbitrary monomial ideal.

3. $\operatorname{reg}\left(\operatorname{in}\left(I^{n}\right)\right) \leq \operatorname{reg}(\operatorname{in}(I)) n$ and $\lim _{n \rightarrow \infty} \frac{\operatorname{reg}\left(\operatorname{in}\left(I^{n}\right)\right)}{n}$ exists if $\operatorname{dim} R / I \leq 1$.

Moreover, the $a$-invariant of the graded rings $G\left(R / I^{n}\right), R / I^{(n)}$ and $R / \operatorname{in}\left(I^{n}\right)$ is bounded by a linear function of $n$ for arbitrary dimension. Note that the Castelnuovo-Mumford regularity is always an upper bound for the $a$-invariant.

The paper is divided in three sections.

In Section 1 we first discuss the relationship between the Castelnuovo-Mumford regularity and the reduction number, which is also a measure for the complexity of a graded ring. Then we apply this relationship to study Question 1. Let $r\left(R / I^{n}\right)$ denote the reduction number of the maximal ideal of $R / I^{n}$. We show that $c=$ $\lim _{n \rightarrow \infty} \frac{r\left(R / I^{n}\right)}{n}$ exists and that $r\left(R / I^{n}\right) \leq c n-1$ for all $n$. The answer to Problem 1 in the case $\operatorname{dim} R / I \leq 1$ follows from this fact and Swanson's linear bound for the nilpotency index of primary components of $I^{n}$ (see $[\mathrm{Sw}]$ ). Moreover, we construct examples with $\operatorname{reg}\left(G\left(R / I^{n}\right)\right)=r\left(R / I^{n}\right)$ for all $n \geq 1$ such that $\lim _{n \rightarrow \infty} \frac{r\left(R / I^{n}\right)}{n}$ is any given rational number $\geq 1$.

In Section 2 we study the Castelnuovo-Mumford regularity of the generalized symbolic powers which are defined by taking out those primary components of $I^{n}$ which contain a given ideal $J$. If $\operatorname{dim} R / J \leq 2$, we can bound $\operatorname{reg}\left(I^{(n)}\right)$ by means of $\operatorname{reg}\left(I^{n}\right)$. The first two cases of the above-mentioned results to Question 2 are simple 
consequences of this fact. The case of monomial ideals follows from the fact that the regularity of a monomial ideal is bounded by the degree of the least common multiple of the generators (see $[\mathrm{BH} 2$ and $[\mathrm{HT}]$ ).

In Section 3 we study the initial ideal $\operatorname{in}_{\lambda}(I)$ with respect to an integral weight function $\lambda$ instead of the usual initial ideal in $(I)$ with respect to a term order. We show that the Castelnuovo-Mumford regularity and the reduction number of the quotient ring $R / \operatorname{in}_{\lambda}(I)$ do not change when passing to the generic member of a certain flat family over $k[t]$ of quotient rings of $S$. This relationship allows us to prove the above-mentioned result to Question 3. As a byproduct of our approach, we can apply a recent result of Ein, Lazarsfeld and Smith [ELS] to show that for all $n \geq 0, \operatorname{in}\left(I^{s n}\right) \subseteq \operatorname{in}(I)^{n}$, where $s$ is the codimension of $I$.

Acknowledgement. This paper was written during a visit of Lê Tuân Hoa and Ngô Viêt Trung to the University of Essen im Summer 2000. These authors would like to express their sincere thanks to the Research Group "Arithmetic and Geometry" for generous support and hospitality.

\section{Regularity AND REDUCTION NUMBER}

Let us first recall some facts on the Castelnuovo-Mumford regularity of a graded module and its relationship to the reduction number. We will do it in a general setting (since it will be needed later in this paper).

Let $S=S_{0}\left[x_{1}, \ldots, x_{m}\right]$ be a standard graded algebra over a local ring $\left(S_{0}, \mathfrak{n}\right)$. For convenience we assume that the residue field of $S_{0}$ is infinite. Let $E$ be a finitely graded $S$-module. For every integer $i \geq 0$ we set

$$
a_{i}(E):=\max \left\{r \mid H_{S_{+}}^{i}(E)_{r} \neq 0\right\}
$$

with $a_{i}(E)=-\infty$ if $H_{S_{+}}^{i}(E)=0$, where $H_{S_{+}}^{i}(E)$ denotes the $i$ th local cohomology module of $E$ with respect to the ideal $S_{+}=\bigoplus_{n>0} S_{n}$.

Definition. The Castelnuovo-Mumford regularity of $E$ is defined by

$$
\operatorname{reg}(E)=\max \left\{a_{i}(E)+i \mid i \geq 0\right\} .
$$

Note that $H_{S_{+}}^{i}(E)=0$ for $i>\operatorname{dim} E / \mathfrak{n} E$ (see e.g. [T1], [T2]). We will call $E / \mathfrak{n} E$ the fiber module of $E$.

Remark. Let $J$ be a homogeneous ideal of $S$. Since $H_{S_{+}}^{i}(S)=0$ for $i \neq m$ and $H_{S_{+}}^{i}(J)=0$ for $i>m$, from the exact sequence $0 \longrightarrow J \longrightarrow S \longrightarrow S / J$ we can deduce that $\operatorname{reg}(J)=\operatorname{reg}(S / J)+1$.

Definition. A reduction of $E$ is a graded ideal $J$ generated by forms of degree 1 such that $(J E)_{n}=E_{n}$ for all large $n$. The least integer $n_{0}$ for which $(J E)_{n}=E_{n}$ for all $n>n_{0}$ is called the reduction number of $E$ with respect to $J$. It will be denoted by $r_{J}(E)$. A reduction $J$ of $E$ is minimal if $J$ does not contain any other reduction of $E$. We set

$$
r(E):=\min \left\{r_{J}(E) \mid J \text { is a minimal reduction of } E\right\} .
$$

These notions were originally introduced for graded quotient rings of $S$ (see e.g. [T1,, $\mathrm{V}]$ ). 
Remark. If $S$ is a polynomial ring over a field $k$, then $J=\left(z_{1}, \ldots, z_{d}\right)$ is a minimal reduction of a graded quotient ring $S / I$ if and only if $A=k\left[z_{1}, \ldots, z_{d}\right]$ is a Noether normalization of $S / I$. Moreover, $r_{J}(S / I)$ is the maximum degree of the generators of $S / I$ as a graded $A$-module (see e.g. $[\mathrm{V}]$ ).

The following relationship between regularity and reduction number has been proved for graded quotient rings of $S$. But it can be easily extended to the case of graded modules. We leave it to the reader to check the proof.

Proposition 1.1. T1, Proposition 3.2] Let $d=\operatorname{dim} E / \mathfrak{n} E$. Then

$$
a_{d}(E)+d \leq r(E) \leq \operatorname{reg}(E) .
$$

If $S$ is a polynomial ring over a field, we set $a(E)=a_{d}(E)$ and call it the $a$ invariant of $E$. This invariant plays an important role in local duality, since $-a(E)$ is the initial degree of the canonical module of $E$ (see e.g. [GW], [BH1]).

The reduction number of a graded module is a generalization of the reduction number in the local case. Let $(R, \mathfrak{m})$ be a local ring. For convenience we assume that the residue field $k=R / \mathfrak{m}$ is infinite.

Definition. We call an $\mathfrak{m}$-primary ideal $\mathfrak{q}$ a reduction of $\mathfrak{m}$ if $\mathfrak{m}^{r+1}=\mathfrak{q m}^{r}$. The least integer $r \geq 0$ with this property is called the reduction number of $R$ with respect to $\mathfrak{q}$. It will be denoted by $r_{\mathfrak{q}}(R)$. A reduction of $\mathfrak{m}$ is called minimal if it does not contain any other reduction of $\mathfrak{m}$. We will set

$$
r(R)=\min \left\{r_{\mathfrak{q}}(R) \mid \mathfrak{q} \text { is a minimal reduction of } \mathfrak{m}\right\} .
$$

Let $G(R)=\bigoplus_{n \geq 0} \mathfrak{m}^{n} / \mathfrak{m}^{n+1}$ be the associated graded ring of $R$ with respect to $\mathfrak{m}$. Then $G(R)$ is a standard graded $k$-algebra. There is the following relationship between the reduction numbers of $R$ and $G(R)$.

Lemma 1.2. $r(R)=r(G(R))$.

Proof. For any element $z \in \mathfrak{m} \backslash \mathfrak{m}^{2}$ we denote by $z^{*}$ the initial form of $z$ in $G(R)$. Let $z_{1}, \ldots, z_{d}$ be arbitrary elements in $\mathfrak{m} \backslash \mathfrak{m}^{2}, d=\operatorname{dim} R$. It is known that $(\underline{z})=\left(z_{1}, \ldots, z_{d}\right)$ is a minimal reduction of $\mathfrak{m}$ if and only if $\left(\underline{z}^{*}\right)=\left(z_{1}^{*}, \ldots, z_{d}^{*}\right)$ is a minimal reduction of $G(R)$, and that $r_{(\underline{z})}(R)=r_{\left(\underline{z}^{*}\right)}(G(R))$ [NR. Hence the conclusion is immediate.

The regularity of the associated graded ring $G(R)$ can be characterized as follows.

Theorem 1.3. [T2, Theorem 1.1] Let $(R, \mathfrak{m})$ be a local ring with $d=\operatorname{dim} R$. Let $\mathfrak{q}$ be a minimal reduction of $\mathfrak{m}$. Then the following conditions are equivalent for a fixed integer $r \geq 0$ :

(i) $\operatorname{reg}(G(R))=r$.

(ii) There is a minimal basis $z_{1}, \ldots, z_{d}$ for $\mathfrak{q}$ such that

$$
\left[\left(z_{1}, \ldots, z_{i-1}\right): z_{i}\right] \cap \mathfrak{m}^{r+1}=\left(z_{1}, \ldots, z_{i-1}\right) \mathfrak{m}^{r}, i=1, \ldots, d,
$$

and $r$ is the least integer $\geq r_{\mathfrak{q}}(R)$ with this property.

Remark. If $G(R)$ is a Cohen-Macaulay ring, then the initial forms of $z_{1}, \ldots, z_{d}$ in $G(R)$ form a regular sequence. By $[\mathrm{VV}]$ this implies that $z_{1}, \ldots, z_{d}$ is a regular sequence of $R$ and that $\left(z_{1}, \ldots, z_{i-1}\right) \cap \mathfrak{m}^{n+1}=\left(z_{1}, \ldots, z_{i-1}\right) \mathfrak{m}^{n}$ for all $n$ and $i=1, \ldots, d$. Thus, $\operatorname{reg}(G(R))=r_{\mathfrak{q}}(R)=r(R)$ for any minimal reduction $\mathfrak{q}$ of $\mathfrak{m}$ (cf. [T2, Theorem 6.4]). 
The above results show that the reduction number can be used to estimate the Castelnuovo-Mumford regularity. We shall see that the reduction number in the local case has good asymptotic properties (the same result holds for the graded case, and we leave it to the reader to check it). First, we can always find a generic minimal reduction with the smallest reduction number.

Lemma 1.4. Let $(R, \mathfrak{m})$ be a local ring with $d=\operatorname{dim} R$. For a generic choice of elements $z_{1}, \ldots, z_{d}$ in $\mathfrak{m} \backslash \mathfrak{m}^{2}$, the ideal $\mathfrak{q}=\left(z_{1}, \ldots, z_{d}\right)$ is a minimal reduction of $\mathfrak{m}$ with $r_{\mathfrak{q}}(R)=r(R)$.

Proof. It has been shown in [T3] Lemma 4.2] that for a generic choice of linear forms $z_{1}^{*}, \ldots, z_{d}^{*}$ in the associated graded $\operatorname{ring} G(R)$, the ideal $J=\left(z_{1}^{*}, \ldots, z_{d}^{*}\right)$ is a minimal reduction of $G(R)$ with $r_{J}(G(R))=r(G(R))$. If $z_{1}, \ldots, z_{d}$ are elements in $\mathfrak{m} \backslash \mathfrak{m}^{2}$ whose initial forms in $G(R)$ are $z_{1}^{*}, \ldots, z_{d}^{*}$, then $\mathfrak{q}=\left(z_{1}, \ldots, z_{d}\right)$ is also a minimal reduction of $\mathfrak{m}$ with $r_{\mathfrak{q}}(R)=r_{J}(G(R))$ NR. Hence the conclusion follows from Lemma 1.2.

Theorem 1.5. Let $(R, \mathfrak{m})$ be a local ring. Let $\left\{I_{n}\right\}$ be a filtration of ideals in $R$ with $\operatorname{dim} R / I_{n}=\operatorname{dim} R / I_{1}$ for all $n \geq 0$. Then

(i) $r\left(R / I_{n}\right) \leq\left[r\left(R / I_{1}\right)+1\right] n-1$ for all $n \geq 0$,

(ii) $c=\lim _{n \rightarrow \infty} \frac{r\left(R / I_{n}\right)}{n}$ exists, and $r\left(R / I_{n}\right) \geq c n-1$ for all $n \geq 0$.

Proof. It suffices to show that for all integers $a, b \geq 0$,

$$
r\left(R / I_{a+b}\right) \leq r\left(R / I_{a}\right)+r\left(R / I_{b}\right)+1 .
$$

Indeed, (i) is an immediate consequence of this formula. Further, this formula implies that $c=\lim _{n \rightarrow \infty} \frac{r\left(R / I_{n}\right)+1}{n}$ exists and that $r\left(R / I_{n}\right)+1 \geq c n$ for all $n \geq 0$ (see the remark below), hence (ii).

To prove the above formula we let $d=\operatorname{dim} R / I_{1}, r=r\left(R / I_{a}\right)$ and $s=r\left(R / I_{b}\right)$. Using Lemma 1.4 we can find elements $z_{1}, \ldots, z_{d} \in \mathfrak{m}$ such that $\underline{z}=\left\{z_{1}, \ldots, z_{d}\right\}$ generate minimal reductions of the maximal ideals of the local rings $R / I_{a}$ and $R / I_{b}$ with $r_{(\underline{z})}\left(R / I_{a}\right)=r$ and $r_{(\underline{z})}\left(R / I_{b}\right)=s$. Since $\mathfrak{m}^{r+1}+I_{a}=(\underline{z}) \mathfrak{m}^{r}+I_{a}$ and $\mathfrak{m}^{s+1}+I_{b}=(\underline{z}) \mathfrak{m}^{s}+I_{b}$, we get

$$
\begin{aligned}
\mathfrak{m}^{r+1} & \subseteq(\underline{z}) \mathfrak{m}^{r}+I_{a} \cap \mathfrak{m}^{r+1} \\
\mathfrak{m}^{s+1} & \subseteq(\underline{z}) \mathfrak{m}^{s}+I_{b} \cap \mathfrak{m}^{s+1}
\end{aligned}
$$

From this it follows that

$$
\begin{aligned}
\mathfrak{m}^{r+s+2} & \subseteq\left[(\underline{z}) \mathfrak{m}^{r}+I_{a} \cap \mathfrak{m}^{r+1}\right]\left[(\underline{z}) \mathfrak{m}^{s}+I_{b} \cap \mathfrak{m}^{s+1}\right] \\
& \subseteq(\underline{z}) \mathfrak{m}^{r+s+1}+I_{a+b} .
\end{aligned}
$$

Now, we can conclude that $\mathfrak{m}^{r+s+2}+I_{a+b}=(\underline{z}) \mathfrak{m}^{r+s+1}+I_{a+b}$. Hence $\underline{z}$ generates a minimal reduction of the maximal ideal of the local $\operatorname{ring} R / I_{a+b}$ with $r_{(\underline{z})}\left(R / I_{a+b}\right) \leq$ $r+s+1$. Thus, $r\left(R / I_{a+b}\right) \leq r+s+1=r\left(R / I_{a}\right)+r\left(R / I_{b}\right)+1$.

Remark. Let $\left\{c_{n}\right\}$ be any sequence of real positive numbers with the property

$$
c_{a+b} \leq c_{a}+c_{b}
$$

for all $a, b \geq 0$. Then $c=\lim _{n \rightarrow \infty} \frac{c_{n}}{n}$ exists and $c_{n} \geq c n$ for all $n \geq 0$. Indeed, the sequence $\left\{\frac{c_{2^{n}}}{2^{n}}\right\}$ is monotonic decreasing; hence it has a limit $c \geq 0$. Choose $n$ such 
that $0 \leq \frac{c_{2^{n}}}{2^{n}}-c<\varepsilon$. For any integer $p>\frac{2^{n} c_{1}}{\varepsilon}$ write

$$
p=2^{m_{1}}+\cdots+2^{m_{s}}+2^{m_{s+1}}+\cdots+2^{m_{l}},
$$

where $m_{1}<\cdots<m_{s}<n \leq m_{s+1}<\cdots<m_{l}$. From the condition $c_{a+b} \leq c_{a}+c_{b}$ we can deduce that $\frac{c_{p}}{p}<c+2 \varepsilon$. Set $2^{m_{l}+2}=p+p^{\prime}$. Since $c_{p} \geq c_{p+p^{\prime}}-c_{p^{\prime}}$ and $p^{\prime}>\frac{2^{n} c_{1}}{\varepsilon}$, we have $\frac{c_{p}}{p}>c-8 \varepsilon$. Hence the limit exists. Moreover, for each $n>0$, the sequence $\left\{\frac{c_{2^{l} n}}{2^{l} n}\right\}$ is decreasing and converges to $c$. Hence we get $c_{n} \geq c n$.

Corollary 1.6. Let $(R, \mathfrak{m})$ be a local ring. Let $\left\{I_{n}\right\}$ be a filtration of ideals in $R$ with $\operatorname{dim} R / I_{n}=d$ for a fixed integer $d$ and all $n \geq 0$. Then, for all $n \geq 0$,

$$
a\left(G\left(R / I_{n}\right)\right) \leq\left[r\left(R / I_{1}\right)+1\right] n-d-1 .
$$

Proof. By Proposition 1.1 we have $a\left(G\left(R / I_{n}\right)\right) \leq r\left(G\left(R / I_{n}\right)\right)-d$. But $r\left(G\left(R / I_{n}\right)\right)$ $=r\left(R / I_{n}\right)$ by Lemma 1.2 Hence the conclusion follows from Theorem 1.5 (i).

Let $I$ be an arbitrary ideal in the local ring $R$. According to Theorem 1.5 and Corollary 1.6. the reduction number $r\left(G\left(R / I^{n}\right)\right)$ and the $a$-invariant $a\left(G\left(R / I^{n}\right)\right)$ are bounded by a linear function. On the other hand, each associated graded ring $G\left(R / I^{n}\right)$ can be represented as a quotient ring of the associated graded ring $G(R)$ by a homogeneous ideal, say $I_{n}^{*}$. Since $\left\{I_{n}^{*}\right\}$ is a filtration of ideals in $G(R)$, one may ask whether $\operatorname{reg}\left(G\left(R / I^{n}\right)\right)$ is bounded by a linear function. We can give a positive answer to this question in the case $\operatorname{dim} R / I \leq 1$.

Theorem 1.7. Let $(R, \mathfrak{m})$ be a local ring and $I \subset R$ an ideal with $\operatorname{dim} R / I \leq 1$. Then there exists a constant $c \geq 0$ such that for all $n>0$,

$$
\operatorname{reg}\left(G\left(R / I^{n}\right)\right) \leq c n .
$$

Proof. The statement is trivial if $\operatorname{dim} R / I=0$. Let $\operatorname{dim} R / I=1$. Write $I^{n}=$ $J_{n} \cap Q_{n}$, where $J_{n}$ is the intersection of all primary components whose associated primes are different from $\mathfrak{m}$ and $Q_{n}$ is the $\mathfrak{m}$-primary component in an irredundant primary composition of $I^{n}\left(Q_{n}=R\right.$ if $\mathfrak{m}$ is not an associated prime of $\left.I^{n}\right)$. By $[\mathrm{Sw}$. Theorem 3.4], there is a constant $a$ such that for all $n \geq 0$, there is an irredundant decomposition of $I^{n}$ with $\mathfrak{m}^{a n} \subseteq Q_{n}$. From this it follows that

$$
I^{n}=J_{n} \cap\left(\mathfrak{m}^{a n}+I^{n}\right) .
$$

Let $z \in \mathfrak{m}$ be an element such that $z$ generates a minimal reduction of $R / I^{n}$ with $r_{(z)}\left(R / I^{n}\right)=r\left(R / I^{n}\right)$. We have $J_{n}: z=J_{n}$ because $z$ is a non-zerodivisor on $J_{n}$. Therefore, $I^{n}: z=J_{n} \cap\left[\left(\mathfrak{m}^{a n}+I^{n}\right): z\right]$. Hence

$$
\left(I^{n}: z\right) \cap\left(\mathfrak{m}^{a n}+I^{n}\right)=J_{n} \cap\left(\mathfrak{m}^{a n}+I^{n}\right)=I^{n} .
$$

Applying Theorem 1.3 to $R / I^{n}$ with $\mathfrak{q}=\left(z, I^{n}\right) / I^{n}$, we see that

$$
\operatorname{reg}\left(G\left(R / I^{n}\right)\right) \leq \max \left\{\text { an, } r\left(R / I^{n}\right)\right\} .
$$

By Theorem 1.5(i), $r\left(R / I^{n}\right) \leq[r(R / I)+1] n-1$. Therefore, if we put $c=$ $\max \{a, r(R / I)+1\}$, then $\operatorname{reg}\left(G\left(R / I^{n}\right)\right) \leq c n$ for all $n \geq 0$. 
Remark. If $d:=\operatorname{dim} R / I<\operatorname{dim} R$, we have $\lim _{n \rightarrow \infty} \frac{r\left(R / I^{n}\right)}{n} \geq 1$, and therefore $\lim _{n \rightarrow \infty} \frac{\operatorname{reg}\left(G\left(R / I^{n}\right)\right)}{n} \geq 1$ if it exists. Indeed, let $z_{1}, \ldots, z_{d} \in \mathfrak{m}$ be elements such that $\underline{z}=\left\{z_{1}, \ldots, z_{d}\right\}$ generates a minimal reduction of the maximal ideal of $R / I^{n}$. If $\mathfrak{m}^{n-1} \subseteq(\underline{z}) \mathfrak{m}^{n-2}+I^{n}$, then $\mathfrak{m}^{n-1}=(\underline{z}) \mathfrak{m}^{n-2}$. Hence $(\underline{z})$ is a minimal reduction of $\mathfrak{m}$, a contradiction to the assumption $d<\operatorname{dim} R$. So we have $r_{\underline{z}}\left(R / I^{n}\right) \geq n-1$, which implies that $\lim _{n \rightarrow \infty} \frac{r\left(R / I^{n}\right)}{n} \geq 1$.

Now we will construct an example with $\operatorname{reg}\left(G\left(R / I^{n}\right)\right)=r\left(R / I^{n}\right)$ for all $n \geq 1$ such that $\lim _{n \rightarrow \infty} \frac{r\left(R / I^{n}\right)}{n}$ is any given rational number not less than 1 (cf. $[\mathrm{Cu}]$ ). As a consequence, $r\left(R / I^{n}\right)$ and $\operatorname{reg}\left(G\left(R / I^{n}\right)\right)$ need not to be linear functions for large $n$. According to the result of [CHT], this implies that the Rees algebra of the filtration $\left\{I_{n}^{*}\right\}$ in the associated graded ring $G(R)$ is not Noetherian in this case.

Example. Let $R=k\left[\left[x, y, z_{1}, \ldots, z_{d}\right]\right] /\left(x^{p}-y^{q}\right)$, where $p<q$ are two different prime numbers, and $I=(x)$. Then $\operatorname{dim} R / I^{n}=d$. It is clear that $G\left(R / I^{n}\right)$ is a Cohen-Macaulay ring. Hence $\operatorname{reg}\left(G\left(R / I^{n}\right)\right)=r_{\mathfrak{q}}\left(R / I^{n}\right)=r\left(R / I^{n}\right)$ for any minimal reduction $\mathfrak{q}$ of the maximal ideal of $R / I^{n}$. We will show that $\lim _{n \rightarrow \infty} \frac{r\left(R / I^{n}\right)}{n}=$ $q / p$.

Choose $\mathfrak{q}=\left(z_{1}, \ldots, z_{d}\right)$. Set $A=k\left[\left[t^{p}, t^{q}\right]\right]$. Then

$$
r_{\mathfrak{q}}\left(R / I^{n}\right)=\max \left\{s \mid\left(t^{p}, t^{q}\right)^{s} A \nsubseteq t^{q n} A\right\} .
$$

For each integer $n$ write $n=p r+s$, where $r \geq 0$ and $0 \leq s \leq p-1$. Since $\left(t^{p}\right)^{q r+s}=t^{p q r+p s} \notin\left(t^{q}\right)^{p r+s} A$, we have $r_{\mathfrak{q}}\left(R / I^{n}\right) \geq q r+s$. On the other hand, let $l \geq q r+q s+p-1$. Assume that $u \in\left(t^{p}, t^{q}\right)^{l} A$. Then $u$ is a sum of elements of the form $\left(t^{p}\right)^{a}\left(t^{q}\right)^{b} v$, where $a+b \geq l$ and $v \in A$. Since $\left(t^{q}\right)^{p}=\left(t^{p}\right)^{q}$ and $p<q$, we may assume that $b \leq p-1$ and $a \geq l-(p-1) \geq q r+q s$. Then

$$
\begin{aligned}
\left(t^{p}\right)^{a}\left(t^{q}\right)^{b} v & =\left(t^{p}\right)^{q r+q s}\left(t^{p}\right)^{a-q r-q s}\left(t^{q}\right)^{b} v \\
& =t^{p q r+q s}\left(t^{q}\right)^{p s-s}\left(t^{p}\right)^{a-q r-q s}\left(t^{q}\right)^{b} v \\
& \in\left(t^{q}\right)^{p r+s} A .
\end{aligned}
$$

Hence $u \in t^{n} A$ and $r_{\mathfrak{q}}\left(R / I^{n}\right)<q r+q s+p-1$. Together with the inequality $q r+s \leq r_{\mathfrak{q}}\left(R / I^{n}\right)$, this implies that

$$
\lim _{n \rightarrow \infty} \frac{r\left(R / I^{n}\right)}{n}=\lim _{n \rightarrow \infty} \frac{r_{\mathfrak{q}}\left(R / I^{n}\right)}{n}=\frac{q}{p} .
$$

Using the argument above, we can eventually compute $r\left(R / I^{n}\right)$ for given $p, q$ and $n$. For instance, if $p=2$ and $q=3$, then

$$
r\left(R / I^{n}\right)= \begin{cases}3 l & \text { if } n=2 l, \\ 3 l+2 & \text { if } n=2 l+1 .\end{cases}
$$

\section{Asymptotic Regularity of Symbolic POWERS}

Let $R=k\left[x_{1}, \ldots, x_{n}\right]$ be a polynomial ring over a field $k$ and $\mathfrak{m}$ the maximal graded ideal of $R$. Let $I$ be an arbitrary homogeneous ideal of $R$. Given an ideal (or a subset) $J \subset R$, we set

$$
I:\langle J\rangle=\bigcup_{n \geq 0} I: J^{n}
$$


It is easily seen that $I:\langle J\rangle$ is the intersection of all components in a primary decomposition of $I$ whose associated primes do not contain $J$.

Definition. For every integer $n \geq 1$ we call the ideal

$$
I^{(n)}:=I^{n}:\langle J\rangle \text {. }
$$

the $n$th $J$-symbolic power of $I$.

This notion generalizes the (ordinary) symbolic power and the saturated power of $I$. Let $A^{*}(I)$ denote the union of the sets of the associated primes of $I^{n}$ for all $n \geq 0$. It is well-known that $A^{*}(I)$ is a finite set (see e.g. [Mc]) and that $A^{*}(I) \supseteq \operatorname{Min}(I)$, where $\operatorname{Min}(I)$ denotes the set of all minimal associated primes of $I$. If $J$ is the intersection of all primes of $A^{*}(I) \backslash \operatorname{Min}(I)$, then $I^{(n)}$ is the (ordinary) $n$th symbolic power of $I$. If $J=\mathfrak{m}$, then $I^{(n)}$ is the saturation of $I^{n}$.

Remark. The symbolic Rees algebra $\bigoplus_{n \geq 0} I^{(n)} t^{n}$ need not be a Noetherian ring. For instance, if $I$ is the defining ideal of a set of $2 r+1$ points on a rational curve in $\mathbb{P}^{r}, r \geq 2$, then

$$
\operatorname{reg}\left(\left(I^{n}\right)^{s a t}\right)=n+1+\left[\frac{n-2}{r}\right]
$$

is not a linear function CTV, Proposition 7]. Thus, the saturated Rees algebra $\bigoplus_{n \geq 0}\left(I^{n}\right)^{s a t} t^{n}$ is not Noetherian, according to [CHT, Lemma 3.3 and Theorem $3.4]$.

Since $\left\{I^{(n)}\right\}$ is a filtration of homogeneous ideals with $\operatorname{dim} R / I^{(n)}=\operatorname{dim} R / I^{(1)}$, the $a$-invariant of $R / I^{(n)}$ is always bounded by a linear function of $n$.

Proposition 2.1. Let $I$ be an arbitrary homogeneous ideal of $R$, and let $d=$ $\operatorname{dim} R / I^{(1)}$. For all $n \geq 0$ we have

$$
a\left(R / I^{(n)}\right) \leq\left[r\left(R / I^{(1)}\right)+1\right] n-d-1 .
$$

Proof. By Theorem 1.5(i) we have

$$
r\left(R / I^{(n)}\right) \leq\left[r\left(R / I^{(1)}\right)+1\right] n-1 .
$$

Hence the conclusion follows from Proposition 1.1 .

Inspired by Theorem 0.1 and Theorem 0.2 , one may ask whether there is a linear upper bound for $\operatorname{reg}\left(I^{(n)}\right)$. We shall see that this question has a positive answer if $\operatorname{dim} R / J \leq 1$ or if $I$ is a monomial ideal.

Proposition 2.2. Let $J \subset R$ be an ideal with $\operatorname{dim} R / J \leq 1$. For all $n \geq 0$ we have

$$
\operatorname{reg}\left(I^{(n)}\right) \leq \operatorname{reg}\left(I^{n}\right)^{\text {sat }} \leq \operatorname{reg}\left(I^{n}\right) .
$$

Proof. Since $I^{(n)}=\bigcup_{r \geq 0} I^{n}: J^{r}$, the quotient module $I^{(n)} / I^{n}$ is annihilated by some power of $J$. It follows that $\operatorname{dim} I^{(n)} / I^{n} \leq \operatorname{dim} R / J \leq 1$. Therefore, $H_{\mathfrak{m}}^{i}\left(I^{(n)} / I^{n}\right)=0$ for all $i \geq 2$. Now, from the exact sequence

$$
0 \longrightarrow I^{(n)} / I^{n} \longrightarrow R / I^{n} \longrightarrow R / I^{(n)} \longrightarrow 0
$$

we can deduce that there is a surjective map $H_{\mathfrak{m}}^{1}\left(R / I^{n}\right) \longrightarrow H_{\mathfrak{m}}^{1}\left(R / I^{(n)}\right)$, and $H_{\mathfrak{m}}^{i}\left(R / I^{(n)}\right) \cong H_{\mathfrak{m}}^{i}\left(R / I^{n}\right)$ for $i \geq 2$. It follows that $a_{1}\left(R / I^{(n)}\right) \leq a_{1}\left(R / I^{n}\right)$, and $a_{i}\left(R / I^{(n)}\right)=a_{i}\left(R / I^{n}\right)$ for $i \geq 2$. Since $\mathfrak{m}$ is not an associated prime of $I^{(n)}$, we have $H_{\mathfrak{m}}^{0}\left(R / I^{(n)}\right)=0$, and therefore $a_{0}\left(R / I^{(n)}\right)=-\infty$. So we can conclude that

$$
\operatorname{reg}\left(R / I^{(n)}\right)=\max \left\{a_{i}\left(R / I^{(n)}\right)+i \mid i>0\right\} \leq \max \left\{a_{i}\left(R / I^{n}\right)+i \mid i>0\right\} .
$$


On the other hand, since $\left(I^{n}\right)^{\text {sat }} / I^{n}$ is a module of finite length, $H_{\mathfrak{m}}^{i}\left(R /\left(I^{n}\right)^{\text {sat }}\right)=$ $H_{\mathfrak{m}}^{i}\left(R / I^{n}\right)$ for $i>0$. Since $H_{\mathfrak{m}}^{0}\left(R /\left(I^{n}\right)^{\text {sat }}\right)=0$, we have

$$
\operatorname{reg}\left(R /\left(I^{n}\right)^{\text {sat }}\right)=\max \left\{a_{i}\left(R / I^{n}\right)+i \mid i>0\right\} .
$$

So we obtain $\operatorname{reg}\left(R / I^{(n)}\right) \leq \operatorname{reg}\left(R /\left(I^{n}\right)^{\text {sat }}\right) \leq \operatorname{reg}\left(R / I^{n}\right)$, which implies the conclusion.

Theorem 2.3. Let $J \subset R$ be an ideal with $\operatorname{dim} R / J \leq 1$. Then there exists a constant e such that for all $n>0$,

$$
\operatorname{reg}\left(I^{(n)}\right) \leq d(I) n+e .
$$

Proof. This follows from Theorem 0.1 and Proposition 2.2 .

Remark. We may apply Theorem 0.2 to obtain a better bound for $\operatorname{reg}\left(I^{(n)}\right)$.

The following example shows that if $\operatorname{dim} R / J \geq 2$, the inequality $\operatorname{reg}\left(I^{(n)}\right) \leq$ $\operatorname{reg}\left(I^{n}\right)$ does not hold even for monomial ideals.

Example. Let $R=k\left[x_{1}, x_{2}, x_{3}, x_{4}\right]$ and

$$
\begin{aligned}
I & =\left(x_{1}, x_{2}^{2}\right) \cap\left(x_{3}, x_{4}^{2}\right) \cap\left(x_{1}, x_{3}\right) \\
& =\left(x_{1} x_{3}, x_{1} x_{4}^{2}, x_{2}^{2} x_{3}\right) .
\end{aligned}
$$

Let $J=\left(x_{1}, x_{3}\right)$. Then $\operatorname{dim} R / J=2$ and

$$
I^{(n)}=\left(x_{1}, x_{2}^{2}\right)^{n} \cap\left(x_{3}, x_{4}^{2}\right)^{n}
$$

for all $n \geq 1$. Applying the graded version of Theorem 1.3 to the rings $R / I^{n}$ and $R / I^{(n)}$ with $z_{1}=x_{2}+x_{4}, z_{2}=x_{1}+x_{2}+x_{3}$, we can show that $\operatorname{reg}\left(R / I^{n}\right)=3 n-1$ and $\operatorname{reg}\left(R / I^{(n)}\right)=4 n-1$ for all $n \geq 1$. From this it follows that

$$
\operatorname{reg}\left(I^{n}\right)=3 n<4 n=\operatorname{reg}\left(I^{(n)}\right) .
$$

Now we will apply Theorem 2.3 to the ordinary symbolic powers.

Corollary 2.4 (cf. [Ch, Corollary 7]). Let $\operatorname{dim} R / I \leq 2$. Then, for the ordinary symbolic powers of $I$, there exists a constant $e$ such that for all $n \geq 0$,

$$
\operatorname{reg}\left(I^{(n)}\right) \leq d(I) n+e .
$$

Proof. For the ordinary symbolic powers, we can choose $J$ to be the intersection of the primes of $A^{*}(I) \backslash \operatorname{Min}(I)$. From this it follows that $\operatorname{dim} R / J \leq \operatorname{dim} R / I-1 \leq 1$. Hence the conclusion follows from Theorem 2.3

Remark. Chandler proved that if $\operatorname{dim} R / I \leq 2$, then $\operatorname{reg}\left(I^{(n)}\right) \leq \operatorname{reg}(I) n$ for all $n \geq 1$.

Corollary 2.5. Assume that the singular locus of $R / I$ has dimension $\leq 1$. Then, for the ordinary symbolic powers of $I^{n}$, there exists a constant e such that for all $n \geq 0$,

$$
\operatorname{reg}\left(I^{(n)}\right) \leq d(I) n+e .
$$

Proof. Since the singular locus of $R / I$ has dimension $\leq 1,(R / I)_{\mathfrak{p}}$ is a regular local ring for all primes $\mathfrak{p} \supseteq I$ with $\operatorname{dim} R / \mathfrak{p}>1$. For any such $\mathfrak{p}$ it follows that $I_{\mathfrak{p}}$ is a complete intersection and hence $I_{\mathfrak{p}}^{n}$ is an unmixed ideal. As a consequence, $I^{n}$ has no embedded associated primes $\mathfrak{p}$ with $\operatorname{dim} R / \mathfrak{p}>1$. That means $\operatorname{dim} R / \mathfrak{p}<1$ for all primes $\mathfrak{p} \in A^{*}(I) \backslash \operatorname{Min}(I)$. If we choose $J$ to be the intersection of the primes of 
$A^{*}(I) \backslash \operatorname{Min}(I)$, then $\operatorname{dim} R / J \leq 1$. Therefore, the conclusion follows from Theorem 2.3.

In the case $\operatorname{dim} R / J=2$ we can only show that if $d\left(I^{(n)}\right)$, the maximal degree of the defining equations of $I^{(n)}$, is bounded by a linear function, then $\operatorname{reg}\left(I^{(n)}\right)$ is bounded by a linear function, too. Note that if $\operatorname{reg}\left(I^{(n)}\right)$ is bounded by a linear function, then so is $d\left(I^{(n)}\right)$, and that the latter condition can often be checked.

Proposition 2.6. Let $J \subset R$ be an ideal with $\operatorname{dim} R / J=2$. Assume that there is an integer a such that $d\left(I^{(n)}\right) \leq$ an for all $n \geq 1$. Then there is an integer $c$ such that for all $n \geq 1$,

$$
\operatorname{reg}\left(I^{(n)}\right) \leq c n
$$

Proof. From the proof of Proposition 2.2 we see that $a_{0}\left(R / I^{(n)}\right)=-\infty$ and

$$
a_{1}\left(R / I^{(n)}\right) \leq \max \left\{a_{1}\left(R / I^{n}\right), a_{2}\left(I^{(n)} / I^{n}\right)\right\} .
$$

Moreover, since $\operatorname{dim} I^{(n)} / I^{n} \leq \operatorname{dim} R / J=2$, we have $a_{i}\left(I^{(n)} / I^{n}\right)=0$ for $i \geq 3$. From this it follows that $a_{2}\left(R / I^{(n)}\right) \leq a_{2}\left(R / I^{n}\right)$ and $a_{i}\left(R / I^{(n)}\right) \leq a_{i}\left(R / I^{n}\right)$ for $i \geq 3$. Thus, it suffices to show that $a_{2}\left(I^{(n)} / I^{n}\right)$ is bounded by a linear function.

Write $I^{n}=I^{(n)} \cap Q_{n}$, where $Q_{n}$ is the intersection of all components of a primary decomposition of $I^{n}$ whose associated primes contain $J$. Then

$$
I^{(n)} / I^{n} \cong\left(I^{(n)}+Q_{n}\right) / Q_{n} .
$$

By [Sw, Theorem 3.4] we may assume that $J^{b n} \subseteq Q_{n}$ for a fixed integer $b \geq 1$. Choose $z_{1}, z_{2} \in R_{1}$ such that $\mathfrak{q}=\left(z_{1}, z_{2}\right)$ is a minimal reduction in $R / J^{b n}$ with $r_{\mathfrak{q}}\left(R / J^{b n}\right)=r\left(R / J^{b n}\right)$. Then $r_{\mathfrak{q}}\left(R / J^{b n}\right) \leq[r(R / J)+1] b n-1$ by Theorem 1.5 Put $m=[r(R / J)+1] b n$. Then $R_{m}=\left(z_{1}, z_{2}, I^{n}\right)_{m}$. Since $d\left(I^{(n)}\right) \leq a n$, we have

$$
\left(I^{(n)}\right)_{a n+m}=R_{m}\left(I^{(n)}\right)_{a n}=\left(z_{1}, z_{2}, I^{n}\right)_{m}\left(I^{(n)}\right)_{a n} .
$$

From this it follows that $\left(I^{(n)} / I^{n}\right)_{a n+m}=\left[\left(z_{1}, z_{2}\right)\left(I^{(n)} / I^{n}\right)\right]_{a n+m}$. Hence $\mathfrak{q}$ is also a minimal reduction of $I^{(n)} / I^{n}$ with $r_{\mathfrak{q}}\left(I^{(n)} / I^{n}\right) \leq a n+m-1$. Thus, $r\left(I^{(n)} / I^{n}\right) \leq$ $a n+m-1$. By Proposition 1.1, this implies that $a_{2}\left(I^{(n)} / I^{n}\right) \leq a n+m-3$. Hence $a_{2}\left(I^{(n)} / I^{n}\right)$ is bounded by a linear function, as required.

Corollary 2.7. Let $\operatorname{dim} R / I=3$. Let $I^{(n)}$ be the ordinary nth symbolic power of I. Assume that there is an integer a such that $d\left(I^{(n)}\right) \leq$ an for all $n \geq 1$. Then there is an integer $c$ such that for all $n \geq 1$,

$$
\operatorname{reg}\left(I^{(n)}\right) \leq c n .
$$

Proof. For the ordinary symbolic powers, we can choose $J$ to be the intersection of the primes of $A^{*}(I) \backslash \operatorname{Min}(I)$. Hence the assumption $\operatorname{dim} R / I=3 \mathrm{implies} \operatorname{dim} R / J \leq$ 2 .

In the following we will show that $\operatorname{reg}\left(I^{(n)}\right)$ is bounded by a linear function for all monomial ideals $I$ and arbitrary ideals $J \supseteq I$. The proof is based on a result of Bruns and Herzog which says that the regularity of a monomial ideal $I$ is bounded by the least common multiple of the generating monomials of $I$ [BH2, Theorem 3.1]. We shall need the following simple observation. 
Lemma 2.8. Let $I$ be an arbitrary monomial ideal. Let $f$ and $f^{\prime}$ be the least common multiples of the generating monomials of $I$ and $I:\langle J\rangle$, respectively. Then $f^{\prime}$ is a divisor of $f$.

Proof. Let $f=x_{1}^{a_{1}} \cdots x_{m}^{a_{m}}$. Then $x_{i}^{a_{i}}$ is the largest power of $x_{i}$ which appears in a generating monomial of $I, i=1, \ldots, m$. Using the formula

$$
\left(I^{\prime}, g_{1} g_{2}\right)=\left(I^{\prime}, g_{1}\right) \cap\left(I^{\prime}, g_{2}\right),
$$

where $I^{\prime}$ is a monomial ideal and $g_{1}$ and $g_{2}$ are monomials which have no common divisor, we can find a primary decomposition of $I$ such that $x_{i}^{a_{i}}$ is the largest exponent of $x_{i}$ which occurs as a generator of some primary component. The ideal $I:\langle J\rangle$ is obtained from this decomposition by deleting those primary components whose associated primes contain $J$. Thus, the exponent of $x_{i}$ in every generating monomial of $I:\langle J\rangle$ must be less than or equal to $a_{i}$. Hence $f$ is a common multiple of the generating monomials of $I:\langle J\rangle$.

Theorem 2.9. Let $I$ and $J$ be arbitrary monomial ideals. Let $f$ be the least common multiple of the generating monomials of $I$. Then, for all $n \geq 0$,

$$
\operatorname{reg}\left(I^{(n)}\right) \leq(\operatorname{deg} f) n-\operatorname{ht} I+1 .
$$

Proof. We first note that $I^{(n)}$ is the intersection of some primary components of $I^{n}$. Let $g_{n}$ denote the least common multiple of the generating monomials of $I^{(n)}$. It has been shown in [HT, Theorem 3.1] (cf. [BH2, Theorem 3.1] for a weaker form) that

$$
\operatorname{reg}\left(I^{(n)}\right) \leq \operatorname{deg} g_{n}-\operatorname{ht} I^{(n)}+1 \leq \operatorname{deg} g_{n}-\operatorname{ht} I+1 .
$$

If we denote by $f_{n}$ the least common multiple of the generators of $I^{n}$, then $\operatorname{deg} g_{n} \leq$ $\operatorname{deg} f_{n}$ by Lemma 2.8. On the other hand, since $f^{n}$ is a common multiple of the generating monomials of $I^{n}, f_{n}$ is a divisor of $f^{n}$. Therefore, $\operatorname{deg} f_{n} \leq \operatorname{deg} f^{n}=$ $(\operatorname{deg} f) n$. Hence $\operatorname{reg}\left(I^{(n)}\right) \leq(\operatorname{deg} f) n-\operatorname{ht} I+1$.

Remark. The previous example shows that we do not always have the inequality $\operatorname{reg}(I:\langle J\rangle) \leq \operatorname{reg}(I)$ for monomial ideals $I$ and $J$.

\section{Asymptotic REgUlARITy OF INITIAL IDEALS}

Let $R=k\left[x_{1}, \ldots, x_{m}\right]$ be a polynomial ring over a field $k$, and $\mathfrak{m}$ the maximal graded ideal of $R$. Let $I$ be an arbitrary homogeneous ideal of $R$. Let in $(I)$ denote the initial ideal of $I$ with respect to a term order of $R$. The aim of this section is to study the regularity of the initial ideal in $\left(I^{n}\right)$. We will do it in a more general setting by investigating the initial ideals of a weight order.

Given a linear map $\lambda: \mathbb{Z}^{m} \rightarrow \mathbb{Z}$, we can define a weight order on the monomials of $R$. Let $\operatorname{in}_{\lambda}(I)$ denote the initial ideal of $I$ with respect to this monomial order. It can be shown that $\operatorname{in}(I)=\operatorname{in}_{\lambda}(I)$ for a suitable choice of the integral weight function $\lambda$ which depends on $I$ (see e.g. [E, p. 327]). The ideal $\operatorname{in}_{\lambda}(I)$ can be obtained as follows.

Let $R[t]$ be a polynomial ring over $R$ in one variable $t$. For any $g \in R[t]$, $g=\sum_{i} a_{i} u_{i}$, where the $u_{i}$ are monomials and $0 \neq a_{i} \in k$, we set $b(g)=\max \lambda\left(u_{i}\right)$ and define $g^{*} \in R[t]$ as follows:

$$
g^{*}:=t^{b(g)} g\left(t^{-\lambda\left(x_{1}\right)} x_{1}, \ldots, t^{-\lambda\left(x_{r}\right)} x_{m}\right) .
$$


We denote by $I^{*}$ the ideal of $R[t]$ generated by $\left\{g^{*} \mid g \in I\right\}$. It is known that $t$ is a non-zerodivisor modulo $I^{*}$ and that $R[t] /\left(I^{*}, t\right) \cong R / \operatorname{in}_{\lambda}(I)$.

In order to study the regularity of $\operatorname{in}_{\lambda}(I)$ we consider the extension of $I^{*}$ in the localization $S=R \otimes k[t]_{(t)}$. We will view $S$ as a standard graded algebra over the local ring $k[t]_{(t)}(\operatorname{deg} t=0)$. Let $\widetilde{I}=I^{*} S$. Then $t$ is still a non-zerodivisor modulo $\widetilde{I}$, and

$$
S /(\widetilde{I}, t) \cong R / \operatorname{in}_{\lambda}(I) .
$$

Since $\widetilde{I}$ and $\operatorname{in}_{\lambda}(I)$ are homogeneous ideals with respect to this grading, we can define the regularity and the reduction number for these ideals as in Section 1.

Proposition 3.1. With the above notations we have

$$
\operatorname{reg}\left(\operatorname{in}_{\lambda}(I)\right)=\operatorname{reg}(\widetilde{I}) .
$$

Proof. From the exact sequence $0 \longrightarrow S / \widetilde{I} \stackrel{t}{\longrightarrow} S / \widetilde{I} \longrightarrow R /$ in $_{\lambda}(I) \longrightarrow 0$ we get the long exact sequence

$$
\cdots \longrightarrow H_{S_{+}}^{i}(S / \widetilde{I}) \stackrel{t}{\longrightarrow} H_{S_{+}}^{i}(S / \widetilde{I}) \longrightarrow H_{\mathfrak{m}}^{i}\left(R / \operatorname{in}_{\lambda}(I)\right) \longrightarrow H_{S_{+}}^{i+1}(S / \widetilde{I}) \longrightarrow \cdots,
$$

where the homomorphisms are all of degree 0. It follows that $a_{i}\left(R / \operatorname{in}_{\lambda}(I)\right) \leq$ $\max \left\{a_{i}(S / \widetilde{I}), a_{i+1}(S / \widetilde{I})\right\}$. Hence

$$
\begin{aligned}
\operatorname{reg}\left(\operatorname{in}_{\lambda}(I)\right) & =\max \left\{a_{i}\left(R / \operatorname{in}_{\lambda}(I)\right)+i \mid i \geq 0\right\} \\
& \leq \max \left\{a_{i}(S / \widetilde{I})+i \mid i \geq 0\right\}=\operatorname{reg}(S / \widetilde{I}),
\end{aligned}
$$

which implies $\operatorname{reg}\left(\operatorname{in}_{\lambda}(I)\right) \leq \operatorname{reg}(\widetilde{I})$.

To prove the converse, let $a_{i}=a_{i}\left(R / \operatorname{in}_{\lambda}(I)\right)$. Then $H_{\mathfrak{m}}^{i}\left(R / \operatorname{in}_{\lambda}(I)\right)_{n}=0$ for $n>a_{i}$. Therefore, $t H_{S_{+}}^{i}(S / \widetilde{I})_{n}=H_{S_{+}}^{i}(S / \widetilde{I})_{n}$ for $n>a_{i}$. Since $H_{S_{+}}^{i}(S / \widetilde{I})_{n}$ is a finitely generated module over the local ring $k[t]_{(t)}$, this implies $H_{S_{+}}^{i}(S / \widetilde{I})_{n}=0$ by Nakayama's lemma. Thus, $a_{i}(S / \widetilde{I}) \leq a_{i}$. Hence

$$
\operatorname{reg}(S / \widetilde{I}) \leq \max \left\{a_{i}+i \mid i \geq 0\right\}=\operatorname{reg}\left(R / \operatorname{in}_{\lambda}(I)\right),
$$

which implies $\operatorname{reg}(\widetilde{I}) \leq \operatorname{reg}\left(\operatorname{in}_{\lambda}(I)\right)$.

Lemma 3.2. $r\left(R / \operatorname{in}_{\lambda}(I)\right)=r(S / \widetilde{I})$.

Proof. First we note that $R / \operatorname{in}_{\lambda}(I)=S /(\widetilde{I}, t)$ is the fiber ring of $S / \widetilde{I}$. Therefore, a reduction $J$ of $S / \widetilde{I}$ is minimal if and only if $J$ is a minimal reduction of $R / \operatorname{in}_{\lambda}(I)$. Moreover, we have $r_{J}(S / \widetilde{I})=r_{J}\left(R / \operatorname{in}_{\lambda}(I)\right)$. From this it follows that $r\left(R / \operatorname{in}_{\lambda}(I)\right)=r(S / \widetilde{I})$.

According to Proposition 3.1, to estimate $\operatorname{reg}\left(\operatorname{in}_{\lambda}(I)\right)$ we only need to estimate $\operatorname{reg}(\tilde{I})$. To compute the local cohomology modules of $S / \tilde{I}$ we shall need the following observation.

Lemma 3.3. $a_{0}(S / \tilde{I}) \leq a_{0}(R / I)$.

Proof. We have $H_{\mathfrak{m}}^{0}(R / I)=I:\langle\mathfrak{m}\rangle / I$ and, since $S_{+}$is generated by $\mathfrak{m}, H_{S_{+}}^{0}(S / \widetilde{I})=$ $\widetilde{I}:\langle\mathfrak{m}\rangle / \widetilde{I}$. So we have to prove that $[\widetilde{I}:\langle\mathfrak{m}\rangle / \widetilde{I}]_{n}=0$ if $[I:\langle\mathfrak{m}\rangle / I]_{n}=0$.

Let $T=R\left[t, t^{-1}\right]$. Then $T$ can be viewed as a standard graded algebra over $k\left[t, t^{-1}\right]$. Let $\phi$ be thegraded automorphism of $T$ determined by $\phi\left(x_{i}\right)=t^{-\lambda\left(x_{i}\right)} x_{i}$, 
$i=1, \ldots, m$. Let $\phi(I)$ denote the ideal of $R\left[t, t^{-1}\right]$ generated by the elements $\phi(g)$, $g \in I$. It is easy to check that

$$
I^{*}=\phi(I) \cap R[t] .
$$

From this it follows that

$$
I^{*}:\langle\mathfrak{m}\rangle=(\phi(I):\langle\mathfrak{m}\rangle) \cap R[t]=\phi(I:\langle\mathfrak{m}\rangle) \cap R[t] .
$$

If we consider $R[t]$ as a standard graded algebra over $k[t]$, then these formulae also hold for the corresponding graded pieces. Thus, if $(I:\langle\mathfrak{m}\rangle)_{n}=I_{n}$, then $\left(I^{*}:\langle\mathfrak{m}\rangle\right)_{n}=\left(I^{*}\right)_{n} ;$ hence $(\widetilde{I}:\langle\mathfrak{m}\rangle)_{n}=\widetilde{I}_{n}$.

Now, we will use the above relationships between $\operatorname{in}_{\lambda}\left(I^{n}\right)$ and $\widetilde{I^{n}}$ to study the asymptotic regularity of $\operatorname{in}_{\lambda}\left(I^{n}\right)$.

Theorem 3.4. Let $\operatorname{dim} R / I \leq 1$. Then

(i) $\operatorname{reg}\left(\operatorname{in}_{\lambda}\left(I^{n}\right)\right) \leq \operatorname{reg}\left(\operatorname{in}_{\lambda}(I)\right) n$ for all $n \geq 0$,

(ii) $c=\lim _{n \rightarrow \infty} \frac{\operatorname{reg}\left(\operatorname{in}_{\lambda}\left(I^{n}\right)\right)}{n}$ exists with $c \leq \max \left\{d(I), r\left(R / \operatorname{in}_{\lambda}(I)\right)+1\right\}$.

Proof. By Proposition 3.1 and Lemma 3.2 we may replace $\operatorname{in}_{\lambda}\left(I^{n}\right)$ by $\widetilde{I^{n}}$ in the above statements. Note that $\left.S / \widetilde{I^{n}}, t\right) \cong R / \operatorname{in}_{\lambda}\left(I^{n}\right)$ is the fiber ring of $S / \widetilde{I^{n}}$. Since $\operatorname{dim} R / \operatorname{in}_{\lambda}\left(I^{n}\right)=\operatorname{dim} S / I^{n}=\operatorname{dim} S / I \leq 1, a_{i}\left(S / \widetilde{I^{n}}\right)=0$ for $i>2$. Therefore, using Proposition 1.1 we obtain the formula

$$
\operatorname{reg}\left(S / \widetilde{I^{n}}\right)=\max \left\{a_{0}\left(S / \widetilde{I^{n}}\right), r\left(S / \widetilde{I^{n}}\right)\right\}
$$

By Lemma 3.3(ii) we have $a_{0}\left(S / \widetilde{I^{n}}\right) \leq a_{0}\left(R / I^{n}\right) \leq \operatorname{reg}\left(R / I^{n}\right)$. On the other hand, $\operatorname{reg}\left(I^{n}\right) \leq \operatorname{reg}(I) n$ by $\mathrm{Ch}$ and [GGP. From this it follows that $\operatorname{reg}\left(R / I^{n}\right) \leq$ $[\operatorname{reg}(R / I)+1] n-1$. Since $R / \operatorname{in}_{\lambda}(I)$ is a flat deformation of $R / I$ [E] Theorem 15.17], $\operatorname{reg}(R / I) \leq \operatorname{reg}\left(R / \operatorname{in}_{\lambda}(I)\right)=\operatorname{reg}(S / \tilde{I})$. Therefore,

$$
a_{0}\left(S / \widetilde{I^{n}}\right) \leq[\operatorname{reg}(S / \tilde{I})+1] n-1 .
$$

Since $\left\{\widetilde{I^{n}}\right\}$ is a filtration of ideals with $\left.\operatorname{dim} S / \widetilde{I^{n}}, t\right)=\operatorname{dim} R / I$ for all $n \geq 0$, we can apply the graded version of Theorem 1.5 (i) and obtain

$$
r\left(S / \widetilde{I^{n}}\right) \leq[r(S / \tilde{I})+1] n-1 .
$$

On the other hand, $r(S / \tilde{I}) \leq \operatorname{reg}(S / \tilde{I})$ by Proposition 1.1. Therefore, we also have

$$
r\left(S / \widetilde{I^{n}}\right) \leq[\operatorname{reg}(S / \tilde{I})+1] n-1 .
$$

Summing up, we get $\operatorname{reg}\left(S / \widetilde{I^{n}}\right) \leq[\operatorname{reg}(S / \tilde{I})+1] n-1$, which implies (i).

By the above arguments we have

$$
\operatorname{reg}\left(S / \widetilde{I^{n}}\right) \leq \max \left\{\operatorname{reg}\left(R / I^{n}\right), r\left(S / \widetilde{I^{n}}\right)\right\} \leq \operatorname{reg}\left(S / \widetilde{I^{n}}\right) .
$$


This implies $\operatorname{reg}\left(S / \widetilde{I^{n}}\right)=\max \left\{\operatorname{reg}\left(R / I^{n}\right), r\left(S / \widetilde{I^{n}}\right)\right\}$. By Theorem 0.1 we have $\lim _{n \rightarrow \infty} \frac{\operatorname{reg}\left(R / I^{n}\right)}{n} \leq d(I)$. By Theorem 1.5] $c_{1}=\lim _{n \rightarrow \infty} \frac{r\left(S / \widetilde{I^{n}}\right)}{n}$ exists with $c_{1} \leq$ $r(S / \widetilde{I})+1$. So we get

$$
\begin{aligned}
\lim _{n \rightarrow \infty} \frac{\operatorname{reg}\left(S / \widetilde{I^{n}}\right)}{n} & =\max \left\{\lim _{n \rightarrow \infty} \frac{\operatorname{reg}\left(R / I^{n}\right)}{n}, \lim _{n \rightarrow \infty} \frac{r\left(S / \widetilde{I^{n}}\right)}{n}\right\} \\
& \leq \max \{d(I), r(S / \widetilde{I}))+1\},
\end{aligned}
$$

which implies (ii). The proof of Theorem 3.4 is now complete.

We cannot simply translate Theorem 3.4 for initial ideals (with respect to a term order). For, we cannot always find an integral weight function $\lambda$ such that $\operatorname{in}\left(I^{n}\right)=\operatorname{in}_{\lambda}\left(I^{n}\right)$ for all $n \geq 0$.

Theorem 3.5. Let $I$ be a homogeneous ideal with $\operatorname{dim} R / I \leq 1$. Then

(i) $\operatorname{reg}\left(\operatorname{in}\left(I^{n}\right)\right) \leq \operatorname{reg}(\operatorname{in}(I)) n$ for all $n \geq 0$,

(ii) $c=\lim _{n \rightarrow \infty} \frac{\operatorname{reg}\left(\operatorname{in}\left(I^{n}\right)\right)}{n}$ exists with $c \leq \max \{d(I), r(R / \operatorname{in}(I))+1\}$.

Proof. For every $n \geq 0$ we can find an integral weight function $\lambda$ such that $\operatorname{in}\left(I^{r}\right)=$ $\operatorname{in}_{\lambda}\left(I^{r}\right)$ for all $r \leq n$. Therefore, (i) follows from Theorem 3.4(i). The proof for (ii) is similar to the proof for Theorem 3.4(ii). Indeed, we have

$$
\operatorname{reg}\left(S / \widetilde{I^{n}}\right)=\max \left\{\operatorname{reg}\left(R / I^{n}\right), r\left(S / \widetilde{I^{n}}\right)\right\}
$$

By Proposition 3.1 and Lemma 3.2 this implies

$$
\operatorname{reg}\left(R / \operatorname{in}\left(I^{n}\right)\right)=\max \left\{\operatorname{reg}\left(R / I^{n}\right), r\left(R / \operatorname{in}\left(I^{n}\right)\right)\right\} .
$$

Hence the conclusion follows from Theorem 0.1 and Theorem 1.5.

Remark. If $\operatorname{dim} R / I \geq 2$, Sturmfels [St] gave an example showing that the inequality $\operatorname{reg}\left(I^{n}\right) \leq \operatorname{reg}(I) n$ does not hold for monomial ideals. Therefore, Theorem 3.4(i) and Theorem 3.5 (i) do not hold in this case.

We do not know whether $\operatorname{reg}\left(\operatorname{in}\left(I^{n}\right)\right)$ is bounded by a linear function in general. By [BH2, Theorem 3.1] and [HT, Theorem 3.1], this is the case if and only if $d\left(\operatorname{in}\left(I^{n}\right)\right)$ is bounded by a linear function. Apart from the above positive answer for the case $\operatorname{dim} R / I \leq 1$, we can only show that the $a$-invariant of $R / \operatorname{in}\left(I^{n}\right)$ is bounded by a linear function.

Proposition 3.6. Let $I$ be an arbitrary homogeneous ideal of $R$ and $d=\operatorname{dim} R / I$. For all $n \geq 0$ we have

$$
a\left(R / \operatorname{in}\left(I^{n}\right)\right) \leq[r(R / \operatorname{in}(I))+1] n-d-1 .
$$

Proof. Since $\left\{\operatorname{in}\left(I^{n}\right)\right\}$ is a filtration of homogeneous ideals with $\operatorname{dim} R / \operatorname{in}\left(I^{n}\right)=$ $\operatorname{dim} R / I$, we can apply Theorem 1.5 (i) and obtain

$$
r\left(R / \operatorname{in}\left(I^{n}\right)\right) \leq[r(R / \operatorname{in}(I))+1] n-1 .
$$

Hence the conclusion follows from Proposition 1.1.

We shall see that the ideal $\widetilde{I^{n}}$ may be considered as a symbolic power of $\widetilde{I}$. 
Lemma 3.7. $\widetilde{I^{n}}=\widetilde{I}^{n}:\langle t\rangle$ for all $n \geq 0$.

Proof. It suffices to show that $\left(I^{n}\right)^{*}=\left(I^{*}\right)^{n}:\langle t\rangle$. Since $t$ is a non-zerodivisor on $\left(I^{n}\right)^{*}$ and since $\left(I^{n}\right)^{*} \supseteq\left(I^{*}\right)^{n}$, we have $\left(I^{n}\right)^{*}=\left(I^{n}\right)^{*}:\langle t\rangle \supseteq\left(I^{*}\right)^{n}:\langle t\rangle$. Conversely, if $f=f_{1} \cdots f_{n} \in I^{n}$ with $f_{1}, \ldots, f_{n} \in I$, then we can find an integer $a$ such that $t^{a} f^{*}=f_{1}^{*} \cdots f_{n}^{*} \in\left(I^{*}\right)^{n}$. From this it follows that for any element $g \in I^{n}$, we have $t^{a} g^{*} \in\left(I^{*}\right)^{n}$ for all $a$ large enough; hence $g^{*} \in\left(I^{*}\right)^{n}:\langle t\rangle$. So we can conclude that $\left(I^{n}\right)^{*}=\left(I^{*}\right)^{n}:\langle t\rangle$.

The above observation has the following interesting consequence.

Proposition 3.8. Let $I \subset R$ be a homogeneous ideal of codimension s. Then for all $n \geq 0$ we have

$$
\operatorname{in}\left(I^{s n}\right) \subseteq \operatorname{in}(I)^{n} .
$$

Proof. For a fixed natural number $n$ there exists an integral weight function $\lambda$ such that $\operatorname{in}(I)=\operatorname{in}_{\lambda}(I)$ and $\operatorname{in}\left(I^{s n}\right)=\operatorname{in}_{\lambda}\left(I^{s n}\right)$. By Lemma 3.7, $\widetilde{I^{s n}}=\tilde{I}^{s n}:\langle t\rangle$. Since $t$ is a non-zerodivisor modulo $\widetilde{I}$, we have $\tilde{I}^{s n}:\langle t\rangle \subseteq \tilde{I}^{(s n)}$. By a recent result of Ein, Lazarsfeld and Smith [ELS] (see also [HH]), for any ideal $J \subset S$ of codimension $s$ and all $n \geq 0$ we have $\tilde{J}^{(s n)} \subset \tilde{J}^{n}$ for the ordinary symbolic powers. Therefore we get

$$
\widetilde{I^{s n}}=\tilde{I}^{s n}:\langle t\rangle \subseteq \tilde{I}^{(s n)} \subseteq \tilde{I}^{n} .
$$

This implies $\left(\widetilde{I^{s n}}, t\right) \subseteq\left(\tilde{I}^{n}, t\right)$. Hence $\operatorname{in}\left(I^{s n}\right) \subseteq \operatorname{in}(I)^{n}$.

Finally, we will give some examples which show that the initial Rees algebra $\bigoplus_{n \geq 0}$ in $\left(I^{n}\right) t^{n}$ need not be Noetherian.

Example. Let $k$ be any field, $I \subset k[x, y]$ the ideal generated by $x^{3}+y^{3}$ and $x y^{2}$, and let $<$ be any term order with $x>y$. The generators of in $\left(I^{n}\right)$ in degree $3 n$ are $x^{n+2 i} y^{2 n-2 i}$ for $i=0, \ldots, n$. Considering $S$-pairs of the generators of $I^{n}$, we see that in $\left(I^{n}\right)$ has no generators in degree $3 n+1$. But on the other hand $y^{3 n+2}$ is a minimal generator of in $\left(I^{n}\right)$. In fact, $y^{5}=y^{2}\left(x^{3}+y^{3}\right)-x^{2}\left(x y^{2}\right)$ is an element of $I$, and $y^{3 n+2}=y^{3 n-1}\left(x^{3}+y^{3}\right)-x y^{3 n-7}\left(x y^{2}\right)^{3}$ belongs to $I^{n}$, since by the induction hypothesis we may assume that $y^{3 n-1} \in I^{n-1}$ and $y^{3 n-7} \in I^{n-2}$. Now it is easily seen that $y^{3 n+2} t^{n}$ belongs to a minimal set of algebra generators of the Rees algebra $\bigoplus_{n \geq 0} \operatorname{in}\left(I^{n}\right) t^{n}$ ated.

The next example shows that not even $\bigoplus_{n \geq 0} \operatorname{Gin}\left(I^{n}\right) t^{n}$ need be finitely gener-

Example. Let $k$ be a field of characteristic 0 and $R=k\left[x_{1}, \ldots, x_{m}\right]$. Let $I=$ $\left(f_{1}, \ldots, f_{m}\right)$ be an ideal generated by a regular sequence of forms of the same degree $a>1$ in $R$. Since $\operatorname{dim} R / I=0$, we have $\operatorname{reg}\left(R / I^{n}\right)=a\left(R / I^{n}\right)=(n-1) a+m(a-1)$ for all $n>0$. Fix a term order. By extending the field $k$ to $k(u)$, where $u$ is a set of new variables, we may assume that $\operatorname{in}\left(I^{n}\right)=\operatorname{Gin}\left(I^{n}\right)$ for all $n$. Each $\operatorname{Gin}\left(I^{n}\right)$ has a minimal generator of the form $x_{m}^{c_{n}}$. Since $\operatorname{Gin}\left(I^{n}\right)$ is a stable ideal and since $\operatorname{dim} R / I=0$, we have

$$
c_{n}=\operatorname{reg}\left(\operatorname{Gin}\left(I^{n}\right)\right)=\operatorname{reg}\left(I^{n}\right)=(n-1) a+m(a-1)+1 .
$$

Were $\bigoplus_{n \geq 0} \operatorname{Gin}\left(I^{n}\right) t^{n}$ finitely generated, there would exist a number $n_{0}$ such that

$$
\operatorname{Gin}\left(I^{n}\right)=\sum_{n_{1}+\cdots+n_{s}=n, n_{1}, \ldots, n_{s} \leq n_{0}} \operatorname{Gin}\left(I^{n_{1}}\right) \cdots \operatorname{Gin}\left(I^{n_{s}}\right)
$$


for all $n>0$. From this it follows that

$$
c_{n}=\min \left\{c_{n_{1}}+\cdots+c_{n_{s}} \mid n_{1}+\cdots+n_{s}=n, n_{1}, \ldots, n_{s} \leq n_{0}\right\} .
$$

We have $c_{n_{1}}+\cdots+c_{n_{s}}=(n-1) a+s[m(a-1)+1]$. For $n>n_{0}$, we must have $s \geq 2$ for all sequences $n_{1}, \ldots, n_{s} \leq n_{0}$ with $n_{1}+\cdots+n_{s}=n$. So we obtain $c_{n_{1}}+\cdots+c_{n_{s}} \geq(n-1) a+2[m(a-1)+1]>c_{n}$, a contradiction.

\section{REFERENCES}

[BEL] A. Bertram, L. Ein and R. Lazarsfeld, Vanishing theorems, a theorem of Severi, and the equations defining projective varieties, J. Amer. Math. Soc. 4 (1991), no. 3, 587-602. MR 92g:14014

[BH1] W. Bruns and J. Herzog, Cohen-Macaulay rings, Cambridge Univ. Press, Cambridge, 1993. MR 95h:13020

[BH2] W. Bruns and J. Herzog, On multigraded resolutions, Math. Proc. Cambridge Phil. Soc. 118 (1995), 245-275. MR 96g:13013

[CTV] M. Catalisano, N.V. Trung and G. Valla, A sharp bound for the regularity index of fat points in general position, Proc. Amer. Math. Soc. 118 (1993), 717-724. MR 93i:13021

[Ch] K. A. Chandler, Regularity of the powers of an ideal, Commun. Algebra 25 (1997), 37733776. MR 98i: 13040

$[\mathrm{Cu}]$ S. D. Cutkosky, Irrational asymptotic behaviour of Castelnuovo-Mumford regularity, J. Reine Angew. Math. 522 (2000), 93-103.

[CEL] S. D. Cutkosky, L. Ein and R. Lazarsfeld, Positivity and complexity of ideal sheaves, Preprint.

[CHT] S. D. Cutkosky, J. Herzog and N. V. Trung, Asymptotic behaviour of the CastelnuovoMumford regularity, Composition Math. 118 (1999), 243-261. MR 2000f:13037

[ELS] L. Ein, R. Lazarsfeld and K. Smith, Uniform bounds and symbolic powers on smooth varieties, Invent. Math. 144 (2001), 241-252.

[E] D. Eisenbud, Commutative algebra, with a view toward algebraic geometry, Springer, 1994. MR 97a:13001

[EG] D. Eisenbud and S. Goto, Linear free resolutions and minimal multiplicities, J. Algebra 88 (1984), 89-133. MR 85f:13023

[GGP] A. V. Geramita, A. Gimigliano and Y. Pitteloud, Graded Betti numbers of some embedded rational $n$-folds, Math. Ann. 301 (1995), no. 2, 363-380. MR 96f:13022

[GW] S. Goto and K. Watanabe, On graded rings I, J. Math. Soc. Japan 30 (1978), 179-213. MR 81m:13021]

[HH] M. Hochster and C. Huneke, Comparison of symbolic and ordinary powers of ideals, to appear in Invent. Math.

[HT] L. T. Hoa and N. V. Trung, On the Castelnuovo-Mumford regularity and the arithmetic degree of monomial ideals, Math. Z. 229 (1998), 519-537. MR 99k:13034

[K] V. Kodiyalam, Asymptotic behaviour of Castelnuovo-Mumford regularity, Proc. Amer. Math. Soc. 128 (2000), 407-411. MR 2000c:13027

[Mc] S. McAdam, Asymptotic prime divisions, Lecture Notes in Mathematics 1023, Springer, 1983. MR 85f: 13018

[NR] D. G. Northcott and D. Rees, Reductions of ideals in local rings, Proc. Cambridge Philos. Soc. 50 (1954), 145-158. MR 15:596a

[SS] K. Smith and I. Swanson, Linear bounds on growth of associated primes, Comm. Algebra 25 (1997), 3071-3079. MR 98k:13003

[St] B. Sturmfels, Four counterexamples in combinatorial algebraic geometry, J. Algebra 230 (2000), 282-294. MR 2001g:13047

[Sw] I. Swanson, Powers of ideals. Primary decompositions, Artin-Rees lemma and regularity, Math. Ann. 307 (1997), 299-313. MR 97j:13005

[T1] N. V. Trung, Reduction exponent and degree bound for the defining equations of graded rings, Proc. Amer. Math. Soc. 101 (1987), 229-236. MR 89i:13031

[T2] N. V. Trung, The Castelnuovo regularity of the Rees algebra and the associated graded ring, Trans. Amer. Math. Soc. 350 (1998), 2813-2832. MR 98j:13006

[T3] N. V. Trung, Gröbner bases, local cohomology and reduction number, Proc. Amer. Math. Soc. 129 (2001), 9-18. MR 2001c:13042 
[VV] P. Valabrega and G. Valla, Form rings and regular sequences, Nagoya Math. J. 72 (1978), 93-101. MR 80d:14010

[V] W. Vasconcelos, Cohomological degrees of graded modules, Six lectures on commutative algebra (Bellaterra, 1996), 345-392, Progr. Math. 166, Birkhäuser, Basel, 1998. MR 99j:13012

Fachbereich Mathematik, Universität-GHS Essen, 45117 Essen, Germany

E-mail address: juergen.herzog@uni-essen.de

Institute of Mathematics, Box 631, Bò Hô, 10000 Hanoi, Vietnam

E-mail address: Ithoa@hanimath.ac.vn

Institute of Mathematics, Box 631, Bò Hô, 10000 Hanoi, Vietnam

E-mail address: nvtrung@hn.vnn.vn 